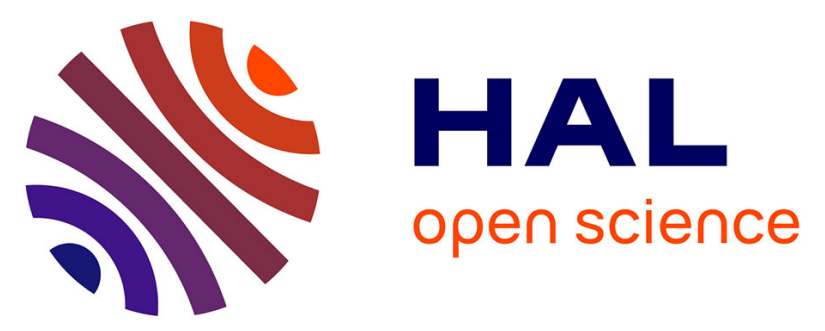

\title{
Corrélations entre les propriétés des dépôts et les conditions de fonctionnement d'une installation de projection plasma: un exemple, l'alumine $\mathbf{y}$
}

Michel Vardelle, Armelle Vardelle, J.L. Besson, Pierre Fauchais

\section{- To cite this version:}

Michel Vardelle, Armelle Vardelle, J.L. Besson, Pierre Fauchais. Corrélations entre les propriétés des dépôts et les conditions de fonctionnement d'une installation de projection plasma: un exemple, l'alumine y. Revue de Physique Appliquée, 1981, 16 (8), pp.425-434. 10.1051/rphysap:01981001608042500 . jpa-00244936

\section{HAL Id: jpa-00244936 \\ https://hal.science/jpa-00244936}

Submitted on 1 Jan 1981

HAL is a multi-disciplinary open access archive for the deposit and dissemination of scientific research documents, whether they are published or not. The documents may come from teaching and research institutions in France or abroad, or from public or private research centers.
L'archive ouverte pluridisciplinaire HAL, est destinée au dépôt et à la diffusion de documents scientifiques de niveau recherche, publiés ou non, émanant des établissements d'enseignement et de recherche français ou étrangers, des laboratoires publics ou privés. 


\title{
Corrélations entre les propriétés des dépôts et les conditions de fonctionnement d'une installation de projection plasma : un exemple, l'alumine $\gamma$
}

\author{
M. Vardelle, A. Vardelle, J. L. Besson et P. Fauchais \\ Equipe de recherche «Céramiques Nouvelles » $(*)$, UER des Ściences, 123, rue A.-Thomas, 87000 Limoges, France
}

(Reçu le 2 octobre 1980, révisé le 28 avril 1981, accepté le 5 mai 1981)

\begin{abstract}
Résumé. - Les propriétés d'un revêtement projeté par plasma dépendent du matériau projeté et des conditions de réalisation du revêtement.

En fait les différents paramètres macroscopiques (nature et caractéristiques des poudres, propriétés du plasma, mode d'injection...) déterminent les propriétés des dépôts par leur action sur les paramètres microscopiques : vitesse et température des particules au moment de l'impact sur le support.

Nous mesurons la vitesse et la température du plasma ainsi que la vitesse et la température des particules pour différentes conditions opératoires de projection. A l'aide de ces mesures, nous étudions l'influence des conditions de projection sur les qualités exigées du revêtement en vue d'optimiser les paramètres de projection.

L'alumine étant une céramique dont les propriétés sont bien connues, nous avons mené les premières études sur ce matériau qui présente en outre la particularité de se trouver en phase métastable après projection.
\end{abstract}

\begin{abstract}
The quality of a plasma sprayed coating is a complex function of sprayed material and operating conditions.

In fact these macroscopic factors can only influence the properties of the coating by controlling the velocity and temperature distribution of the particles at the moment of impact on the substrate. We measure the temperature and the velocity of the plasma jet as the velocity and the temperature of the particles for various operating conditions. With these measurements, we study the influence of the working conditions on the qualities required from the coating, to optimize the spraying process.

Properties of alumina being well known, we have first studied this ceramic, which presents the particularity that, in sprayed alumina deposits, metastable $\gamma-\mathrm{Al}_{2} \mathrm{O}_{3}$ structure is produced.
\end{abstract}

Introduction. - Si le procédé de la projection plasma est développé au niveau industriel depuis les années 1960, les phénomènes qui interviennent sont encore.mal compris du fait de la multiplicité des processus élémentaires qui sont souvent inter-dépendants.

Pour un matériau donné, les propriétés du dépôt dépendent essentiellement de l'énergie cinétique et de la viscosité des particules au moment de l'impact sur la cible, c'est-à-dire de la vitesse et de la température acquises par les particules. Ces deux paramètres sont conditionnés par les transferts de quantité de mouvement et de chaleur entre le plasma et les particules; ils dépendent donc d'une part de facteurs intrinsèques aux poudres utilisées (propriétés thermiques du matériau) et de facteurs extrinsèques (géométrie et morphologie des poudres) et d'autre part de la trajectoire suivie dans le jet de plasma et

$\left(^{*}\right)$ LA CNRS no 320. donc à la fois des conditions d'injection, de la vitesse, de la température et des propriétés du jet de plasma qui découlent des paramètres opératoires de projection (nature et débit du gaz plasmagène, puissance électrique fournie à l'arc...).

Aussi la compréhension des caractéristiques des dépôts requiert la détermination des champs de vitesse et de température du plasma ainsi que la mesure de la vitesse d'injection des poudres, des vitesses et des températures des particules dans le jet de plasma.

A partir de ces mesures il est possible d'optimiser les conditions de fonctionnement d'une installation de projection telle que celle représentée sur la figure 1 pour obtenir des revêtements aux qualités particulières.

Avec les dispositifs de projection, le dépôt d'épaisseur désiré est obtenu par l'empilement de couches successives de particules écrasées et refroidies rapidement sur la cible. 


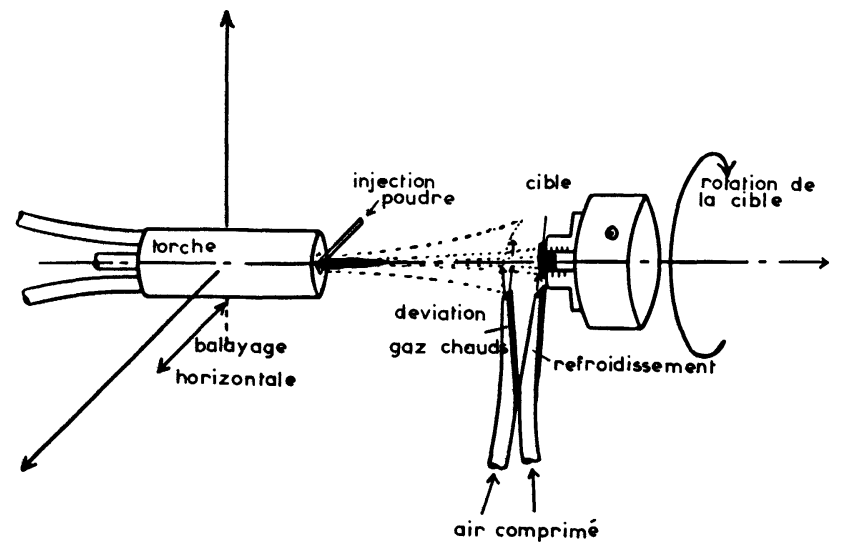

Fig. 1. - Dispositif de la projection plasma.

[Plasma spraying device.]

Pour expliquer les phénomènes rencontrés, il est nécessaire de bien connaître les propriétés du matériau projeté ; c'est pourquoi nous avons choisi l'alumine dont les propriétés physiques et thermiques ainsi que les diverses phases ont été très étudiées.

L'alumine $\gamma$, phase métastable de type spinel, est obtenue sous forme de poudre lors de la calcination à l'air de certains hydrates d'alumine. On l'obtient aussi par projection au chalumeau à plasma d'arc lorsque les conditions de refroidissement sont suffisamment sévères. Cette dernière méthode est la seule conduisant à l'obtention d'échantillons massifs.

En vue d'études ultérieures nous avons cherché, en optimisant les conditions de projection, à obtenir des dépôts denses et contenant la plus faible proportion possible de phase $\alpha$ corindon (la porosité du dépôt dépendant de l'énergie cinétique et de la viscosité des particules au moment du choc sur la cible, et la proportion de phase $\alpha$ de la température des particules et des conditions de leur refroidissement).

1. Le dispositif expérimental de projection et de mesure. - 1.1 LE DISPOSITIF DE PROJECTION. - Le plasma est produit à la pression atmosphérique par un générateur à arc soufflé conçu au laboratoire. $\mathrm{Ce}$ générateur peut fonctionner à l'argon, l'hélium, l'azote et l'hydrogène ou des mélanges de ces divers 'gaz, à condition d'adapter la géométrie des électrodes. Les gaz plasmagènes que nous avons utilisés sont :

- un mélange d'azote à $42 \%$ d'hydrogène soit $37 \mathrm{Nl} / \mathrm{min}$. d'azote et $27 \mathrm{Nl} / \mathrm{min}$. d'hydrogène, le diamètre de tuyère est alors de $6 \mathrm{~mm}$ (Fig. 2),

- un mélange d'argon à $33 \%$ d'hydrogène soit $75 \mathrm{Nl} / \mathrm{min}$. d'argon et $37 \mathrm{Nl} / \mathrm{min}$. d'hydrogène, le diamètre de tuyère est de $8 \mathrm{~mm}$ (Fig. 2).

Les débits respectifs, la nature du gaz, les caractéristiques géométriques des tuyères ont été choisis, comme nous le verrons, afin d'obtenir les meilleures conditions possibles de projection pour les dépôts denses d'alumine $\gamma$.

Afin de ne pas trop compliquer le montage techno-
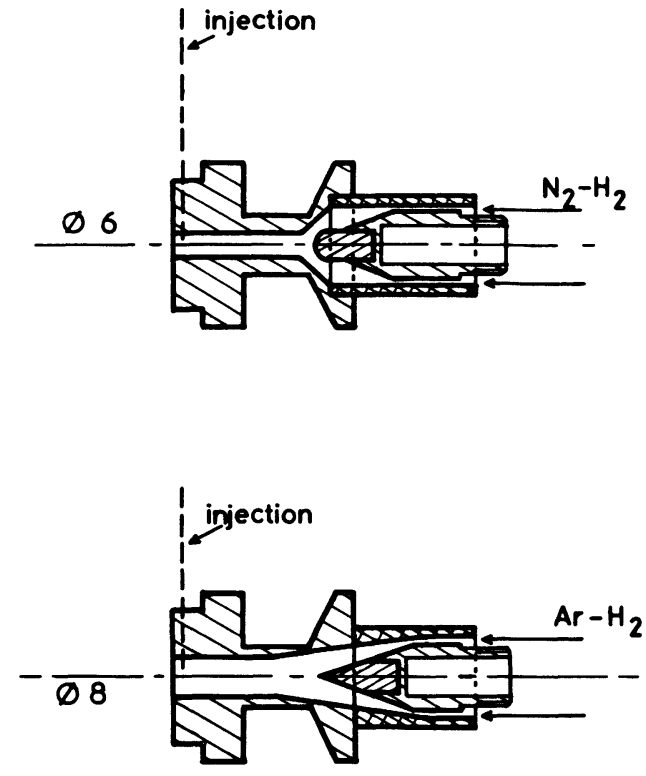

Fig. 2. - Electrodes Ar- $\mathrm{H}_{2}$ et $\mathrm{N}_{2}-\mathrm{H}_{2}$.

[Ar- $\mathrm{H}_{2}$ and $\mathrm{N}_{2}-\mathrm{H}_{2}$ electrodes.]

logique (la tuyère du générateur étant refroidie par circulation d'eau) et de bénéficier cependant de l'injection dans la tuyère c'est-à-dire dans une zone constrictée du plasma pour mieux contrôler la trajectoire des poudres, celles-ci sont introduites à $2 \mathrm{~mm}$ en amont de la sortie de tuyère.

Nous avons utilisé, pour notre étude, des poudres mono-cristallines sous forme corindon, de pureté $99,9 \%$ de granulométrie étroite centrée sur $18 \mu \mathrm{m}$ avec un écart type de $3 \mu \mathrm{m}$ (DGI 5) $\left({ }^{1}\right)$ et des poudres de pureté $99,99 \%$ de granulométrie $18 \pm 10 \mu$ se présentant sous forme d'agrégats de cristallites élémentaires : la poudre GE $1\left(^{2}\right)$ a une structure $\alpha$, la poudre A $125\left({ }^{2}\right)$ contient $70 \%$ de phase $\gamma$ et $30 \%$ de phase $\alpha$. Les tailles des cristallites élémentaires sont de $1,5 \mu \mathrm{m}$ pour GE 1 et $0,01 \mu \mathrm{m}$ pour A 125 (Fig. 3).

Afin d'assurer un refroidissement identique des gouttelettes après leur écrasement et donc de toutes les couches successives déposées, un jet d'air comprimé souffle sur la face avant du dépôt, la buse d'arrivée étant située immédiatement devant la cible. De plus un premier barrage d'air comprimé placé à $15 \mathrm{~mm}$ environ en amont de la cible dévie les gaz chauds pour éviter un échauffement du support [1].

1.2 MeSURE DES VITESSES ET DES TEMPÉRATURES DES PARTICULES. - Nous mesurons la vitesse des particules dans le jet de plasma par des méthodes d'anémométrie laser [2] qui présentent les avantages de ne pas perturber l'écoulement et de permettre l'étude dans

( ${ }^{1}$ Ces poudres nous sont aimablement fournies par M. Yvars du Département de Génie Isotopique, C.E.A. Saclay, France.

$\left({ }^{2}\right)$ Les poudres GE 1 et A 125 sont vendues par La Pierre Synthétique Baikowski, Annecy, France. 


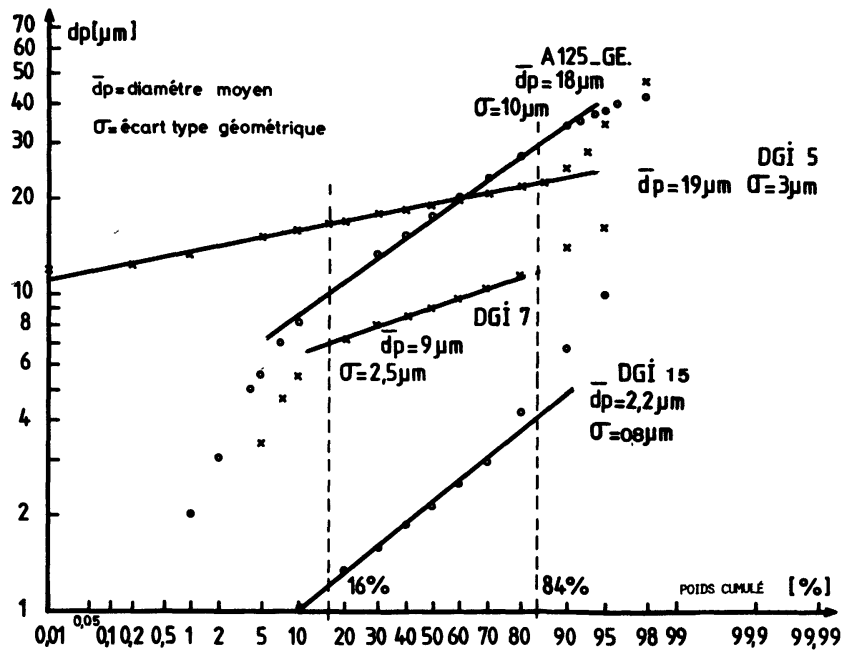

Fig. 3. - Distribution en poids cumulé du diamètre des particules des poudres utilisées.

[Number particle size distribution of the powders used.]

un petit volume de mesure avec une bonne précision. Pour mesurer des vitesses inférieures à $300 \mathrm{~m} / \mathrm{s}$, nous utilisons une méthode de vélocimétrie Doppler laser (V.D.L.) avec un montage de type interférentiel. Les vitesses supérieures à $300 \mathrm{~m} / \mathrm{s}$ sont mesurées par la méthode des deux faisceaux.

En V.D.L. (Fig. 4), nous utilisons un laser à argon ionisé fournissant $800 \mathrm{~mW}$ sur la raie verte $514,5 \mathrm{~nm}$. Un séparateur de faisceaux constitué d'un double prisme assure à la fois la scission du faisceau incident en deux faisceaux d'égale intensité et la focalisation des deux faisceaux obtenus au point de mesure. $\mathrm{Au}$ croisement des faisceaux laser se forme un réseau de franges d'interférences. Lorsqu'une particule traverse le champ d'interférences, elle diffuse une lumière modulée à une fréquence qui est proportionnelle à la composante de la vitesse dans la direction perpendiculaire aux franges et inversement proportionnelle à la distance d'interfrange. La distance d'interfrange est de $100 \mu \mathrm{m}$. L'axe du système optique de réception et l'axe du faisceau laser incident forment un angle de $90^{\circ}$. Si cette géométrie est peu favorable pour le rapport signal sur bruit (elle correspond, en effet, à un minimum de la lumière diffusée par les particules), elle conduit à la meilleure résolution spatiale. Le volume de mesure correspond alors à un cylindre de $600 \mu \mathrm{m}$ de diamètre et de $1100 \mu \mathrm{m}$ de longueur dont l'axe est perpendiculaire au jet de plasma. Notons cependant qu'avec l'interfrange choisie (100 $\mu \mathrm{m}$ afin de réduire le volume de mesure), des vitesses de particules de $300 \mathrm{~m} / \mathrm{s}$ correspondent à une fréquence de $30 \mathrm{MHz}$ limite supérieure de fonctionnement de l'appareillage de détection. Un filtre interférentiel centré sur $514,5 \mathrm{~nm}$ et de $2,5 \AA$ de bande passante est placé devant le photomultiplicateur du système de réception des signaux lumineux. L'analyse des signaux est effectuée par un corrélateur numérique de photons (MALVERN K7023) qui travaille en temps réel en mode auto-corrélation. La rotation du dispositif optique qui sépare en deux faisceaux d'égale intensité le faisceau laser incident, permet la rotation du sys-

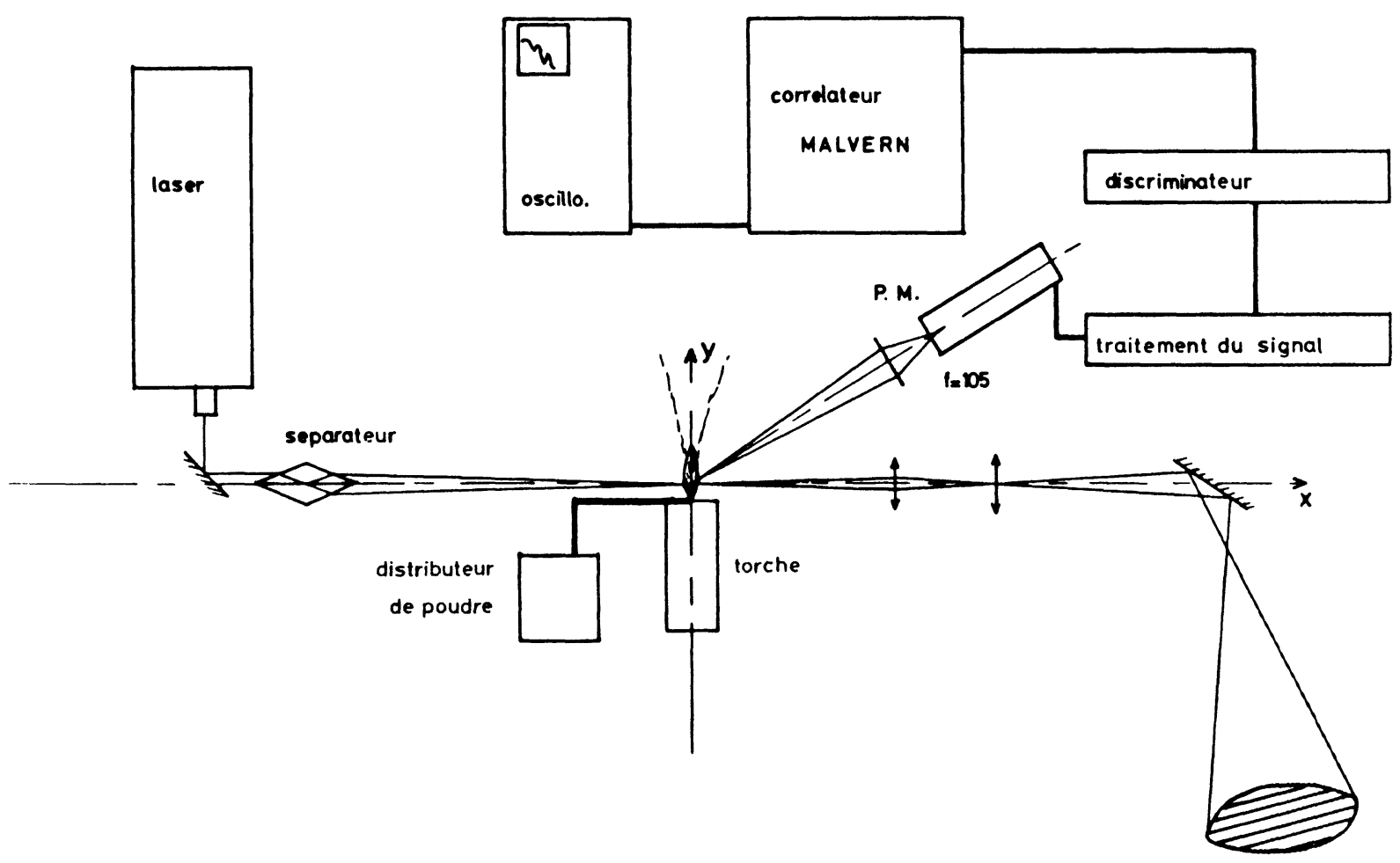

Fig. 4. - Dispositif de mesure de vitesse par A.D.L.

[L.D.A. experimental set up.] 
tème de franges d'interférence et ainsi la détermination des composantes radiale et axiale du vecteur vitesse au point de mesure.

Pour les vitesses supérieures à $300 \mathrm{~m} / \mathrm{s}$, nous utilisons la méthode des deux faisceaux qui consiste à mesurer le temps de transit des particules entre deux points lumineux correspondant aux points de focalisation des deux faisceaux laser.

Après séparation d'un faisceau laser incident $(\lambda=514,5 \mathrm{~nm})$ en deux faisceaux d'égale intensité, une lentille concentre l'énergie de chaque -faisceau laser en un point de focalisation. Le rayon du faisceau au point de focalisation est de $50 \mu \mathrm{m}$ et la distance entre les deux points lumineux est de $400 \mu \mathrm{m}$. Le dispositif de détection de la lumière diffusée comprend deux photomultiplicateurs, un pour chaque faisceau. L'axe du système optique de réception est dans le plan horizontal défini par les faisceaux laser incidents. Cet axe fait un angle de $30^{\circ}$ avec le faisceau incident. Le volume de mesure est un cylindre de $500 \mu \mathrm{m}$ de diamètre et $100 \mu \mathrm{m}$ de longueur. L'analyse des signaux lumineux est effectuée par le corrélateur numérique de photons qui travaille alors en mode cross-corrélation.

Nous déterminons la température de surface des particules à partir de leur rayonnement de surface, d'après une méthode présentée par B. Kruszewska et J. Lesinski [3]. Une lentille forme l'image du jet de plasma sur une fente placée devant un photomultiplicateur dont la photo-cathode est sensible dans le proche infrarouge. Un filtre optique limite le domaine spectral de fonctionnement entre 850 et $1050 \mathrm{~nm}$. Les impulsions électriques issues du photomultiplicateur sont amplifiées et mises en forme, puis analysées par un analyseur multicanal qui donne l'histogramme de la répartition de leurs amplitudes (intertechnique SA 41) (Fig. 5).

Un étalonnage préalable réalisé en simulant des particules de diamètre, de vitesse et de température connus permet de faire la correspondance amplitudetempérature.

Le volume de mesure est un parallélépipède de section $200 \mu \mathrm{m} \times 100 \mu \mathrm{m}$ et de longueur égale à celle d'une corde du jet de plasma.

Pour mesurer le nombre de particules passant en un point donné du jet de plasma, pendant un temps déterminé, nous comptons les impulsions lumineuses dues au passage des particules à travers un faisceau laser focalisé au point de mesure. Le volume de mesure correspond à un cylindre de $100 \mu \mathrm{m}$ de diamètre et de $230 \mu \mathrm{m}$ de longueur, dont l'axe est perpendiculaire au jet de plasma.

1.3 DÉTERMINATION DES CHAMPS DE VITESSE ET DE TEMPÉRATURE DU JET DE PLASMA. - La vitesse du jet de plasma est déterminée par la méthode des deux faisceaux, le jet étant ensemencé avec des particules d'alumine de diamètre inférieur à $3 \mu \mathrm{m}$ introduites dans la chambre d'arc avec le gaz plasma. Le débit de poudre est de l'ordre de $5 \mathrm{~g} / \mathrm{h}$ afin d'éviter la perturbation de l'arc et donc du plasma.

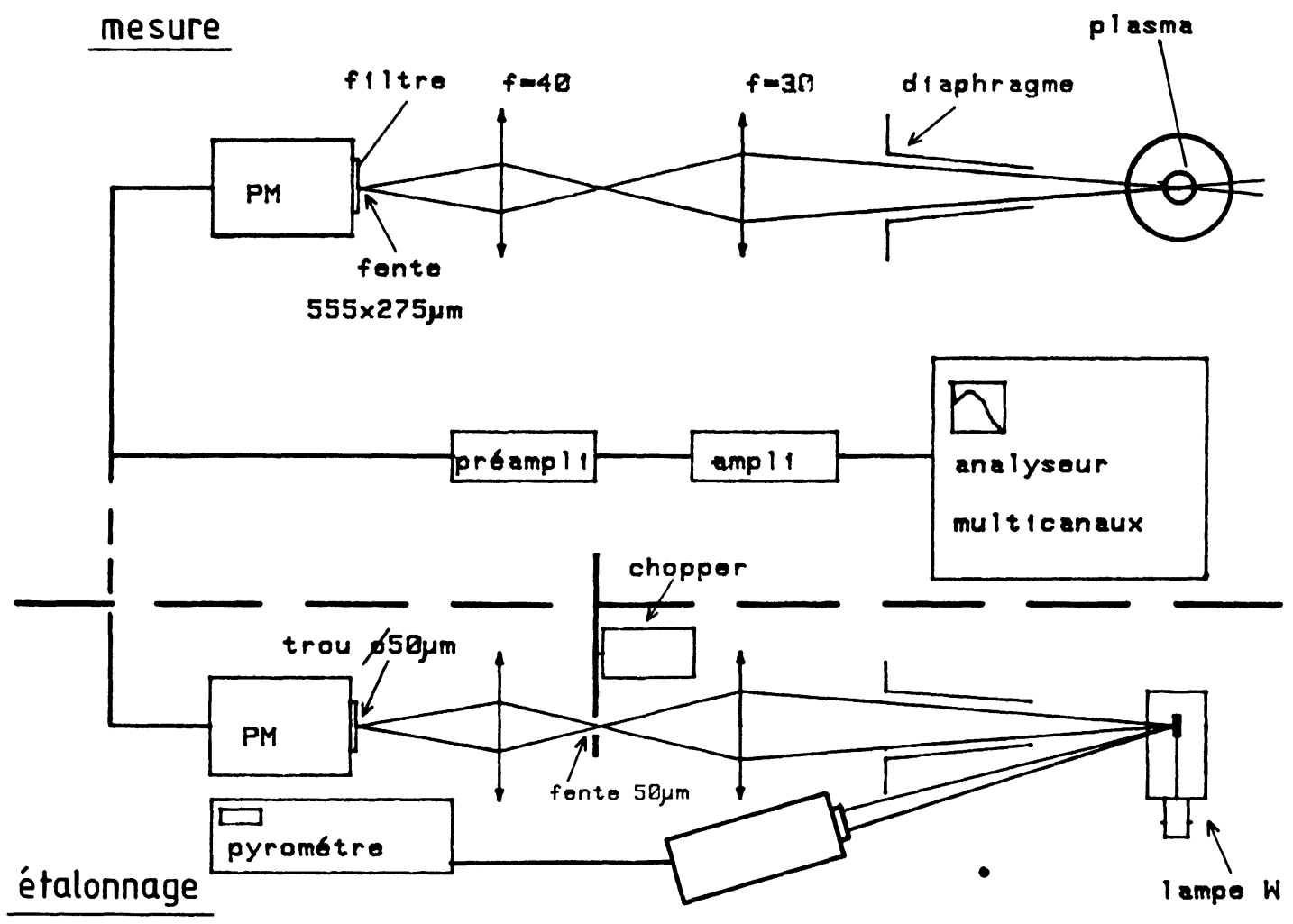

Fig. 5. - Dispositif de mesure de la température des particules.

[Experimental set up of particle temperature measurement.] 
Pour les températures supérieures à $6000 \mathrm{~K}$, le champ des températures du plasma est déterminé par spectroscopie d'émission : le plasma est supposé optiquement mince et en E.T.L. et les températures sont déduites des mesures des coefficients d'émission volumique des raies atomiques, connaissant la composition du plasma en fonction de la température [2].

Pour les gaz chauds, de l'extrémité du panache du jet, les températures sont mesurées par couples thermo-électriques et localisation du point de fusion d'une baguette d'alumine. Les valeurs mesurées sont corrigées de façon à tenir compte du rayonnement sur la sonde et de la conduction thermique dans la sonde.

2. La projection des poudres au chalumeau à plasma. - 2.1 LE TRANSFERT DE CHALEUR ET DE QUANTITÉ DE MOUVEMENT PLASMA-PARTICULE. - La trajectoire d'une particule dans le jet de plasma peut être calculée à partir d'une forme simplifiée de l'équation donnée par Hinze [4], en ne considérant que la force de frottement du fluide sur le grain.

Alors :

$$
m \frac{\mathrm{d} V_{\mathrm{p}}}{\mathrm{d} t}=\frac{1}{2} C_{\mathrm{D}} \rho_{\mathrm{p}} A\left(\mathbf{V}_{\mathrm{p}}-\mathbf{V}_{\mathrm{f}}\right)\left|V_{\mathrm{p}}-V_{\mathrm{f}}\right|
$$

où $V_{\mathrm{p}}$ est la vitesse de la particule, $V_{\mathrm{f}}$ celle du fluide, $m$ la masse de la particule, $\rho_{\mathrm{p}}$ sa masse volumique, $A$ sa section perpendiculaire à la direction de projection et $C_{\mathrm{D}}$ le coefficient de traînée qui ne dépend que du nombre de Reynolds $\operatorname{Re}\left(\operatorname{Re}=\frac{\left(U_{\mathrm{p}}-U_{\mathrm{f}}\right) \rho_{\mathrm{f}} d_{\mathrm{p}}}{n_{\mathrm{f}}}\right)$ où $\rho_{\mathrm{f}}$ est la masse volumique du fluide, $n_{\mathrm{f}}$ sa viscosité et $d_{\mathrm{p}}$ le diamètre de la particule.

Les particules injectées dans le fluide en mouvement sont d'abord accélérées par la force de frottement fluide-particule. Cette force s'annule au point de la trajectoire où les vitesses des particules et du fluide sont égales. Elle devient ensuite une force de freinage. L'accélération des particules dépend de la taille et de la nature des particules et de la nature du fluide. Dans nos conditions de projection l'accélération est de l'ordre de 10000 et $60000 \mathrm{~g}$ tandis que leur décélération varie de 1000 à $20000 \mathrm{~g}$.

Les particules introduites dans le jet de plasma sont chauffées essentiellement par des mécanismes de conduction et convection, ces mécanismes étant souvent accompagnés de phénomènes de changement de phase et de transfert de masse.

Le flux global reçu par la particule est égal à :

$$
q=h_{\mathrm{T}} A\left(T_{\mathrm{f}}-T_{\mathrm{p}}\right)
$$

(le rayonnement du plasma n'étant généralement pas pris en compte) où $h_{\mathrm{T}}$ est le coefficient de transfert de chaleur plasma-particule que l'on détermine à partrr du nombre de Nusselt $N u, N u=h d_{\mathrm{p}} / k_{\mathrm{f}}$, $k_{\mathrm{f}}$ étant la conductivité thermique du fluide, le nombre de Nusselt étant lui-même déterminé à partir de corrélations empiriques.
En raison des forts gradients de température dans la couche limite thermique entourant la particule, on ne peut considérer les propriétés physiques du fluide constantes dans cette couche limite et on démontre [5] qu'il faut considérer la variation globale de ces propriétés au travers de la couche limite thermique en définissant des grandeurs moyennes telles que :

$$
k_{\mathrm{f}}=\frac{1}{T_{\mathrm{f}}-T_{\mathrm{p}}} \int_{T_{\mathrm{p}}}^{T_{\mathrm{f}}} k(T) \mathrm{d} T .
$$

Ainsi les figures 6 et 7 représentent respectivement la conductivité thermique de l'argon, l'azote et l'hydro-

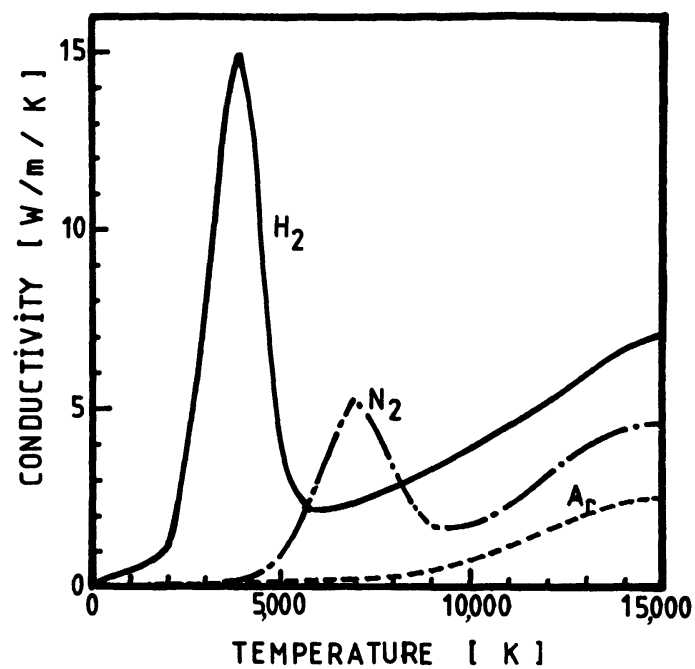

Fig. 6. - Conductivité thermique des gaz $\left(\mathrm{Ar}, \mathrm{N}_{2}, \mathrm{H}_{2}\right)$ en fonction de la température.

[Thermal conductivity of the gases $\left(\mathrm{Ar}, \mathrm{N}_{2}, \mathrm{H}_{2}\right)$ versus temperature.]

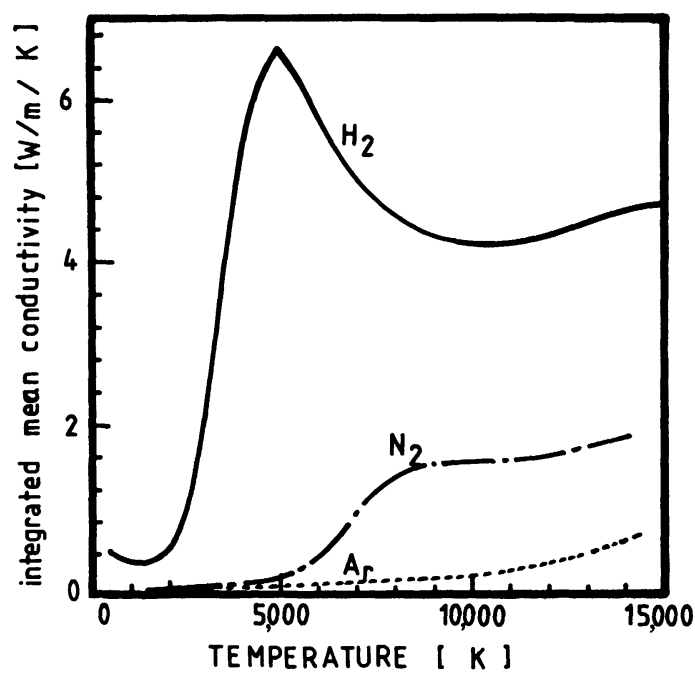

Fig. 7. - Intégrale de la conductivité thermique des gaz $\left(\mathrm{Ar}, \mathrm{N}_{2}\right.$, $\mathrm{H}_{2}$ ) en fonction de la température.

$$
\bar{k}=\frac{1}{(T-300)} \int_{300}^{T} k(t) \mathrm{d} t .
$$

[Integrated thermal conductivity of the gases $\left(\mathrm{Ar}, \mathrm{N}_{2}, \mathrm{H}_{2}\right.$ ) versus temperature.] 
gène et l'intégrale de la conductivité de ces mêmes gaz entre $300 \mathrm{~K}$ et $T$.

Nous constatons que pour une température limite (correspondant à la dissociation du gaz), la conductivité moyenne intégrée augmente dans des proportions notables et que les transferts thermiques qui s'effectuent à des températures supérieures sont augmentés d'autant.

Le comportement thermique d'une particule dans un jet de plasma dépend essentiellement de la nature du matériau, des propriétés du fluide (principalement conductivité thermique et viscosité), de la trajectoire qu'elle suit dans le jet et de son temps de séjour dans les zones chaudes du plasma.

2.2 LE JET DE PLASMA. - Les caractéristiques d'un jet de plasma produit par un générateur à arc soufflé dépendent de la pression, de la nature du gaz utilisé, et des dimensions et des formes des électrodes. Le jet de plasma se caractérise par des gradients radiaux et axiaux de vitesse et de température (donc de propriétés) importants, comme le montrent les figures 8 et 9 qui représentent respectivement les isothermes d'un jet de plasma azote hydrogène à $29 \mathrm{~kW}$ et les cartographies de vitesse des mélanges azotehydrogène et argon-hydrogène que nous utilisons $(P=29 \mathrm{~kW})$.

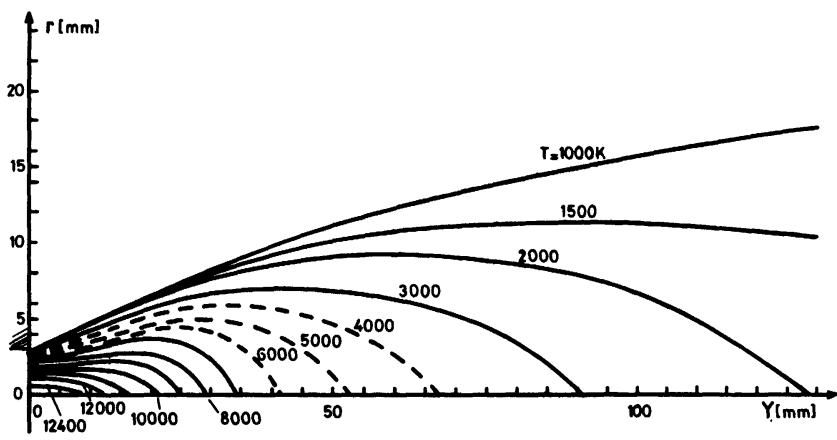

Fig. 8. - Isothermes du jet de plasma azote-hydrogène.

[Temperature distribution in a $\mathrm{N}_{2}-\mathrm{H}_{2}$ plasma jet.]

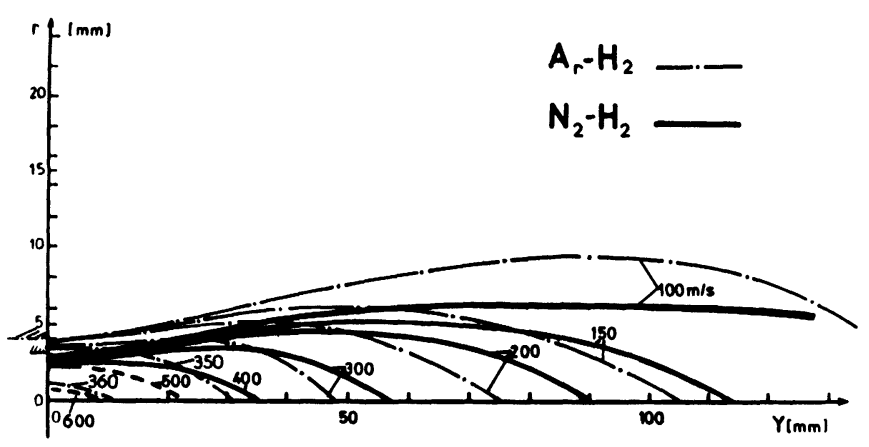

Fig. 9. - Champ de vitesse des jets de plasma argon-hydrogène et azote-hydrogène.

[Velocity distribution in a $\mathrm{N}_{2}-\mathrm{H}_{2}$ and a $\mathrm{Ar}-\mathrm{H}_{2}$ plasma jet.]
Il est possible de distinguer trois zones dans le jet de plasma :

- le dard dont la température est pratiquement constante,

- le panache où la température décroît exponentiellement,

- les gaz chauds qui ont une température inférieure à $2500 \mathrm{~K}$.

Dans le jet de plasma azote-hydrogène on peut constater que les gradients radiaux de température sont très importants jusqu'à $40 \mathrm{~mm}$ de la sortie de tuyère et qu'ils diminuent ensuite rapidement : les profils radiaux de température sont pratiquement plats à $100 \mathrm{~mm}$ de la torche.

Pour les mélanges azote-hydrogène et argon-hydrogène que nous utilisons, nous remarquons que le jet de plasma $\mathrm{Ar}-\mathrm{H}_{2}$ a un rayon supérieur à celui du plasma $\mathrm{N}_{2}-\mathrm{H}_{2}$, ce qui s'explique par les géométries des tuyères utilisées. Egalement il faut noter que le dard et le panache sont plus longs pour le mélange $\mathrm{Ar}-\mathrm{H}_{2}$, comme le montrent les courbes de la figure 10 qui représentent l'évolution axiale de la température du plasma $\mathrm{Ar}-\mathrm{H}_{2}$ et $\mathrm{N}_{2}-\mathrm{H}_{2}$ le long de l'axe de la torche.

$\mathrm{Du}$ fait du rayonnement très intense du plasma dans le dard du jet, il ne nous est pas possible de mesurer la vitesse à moins de $20 \mathrm{~mm}$ de la sortie de

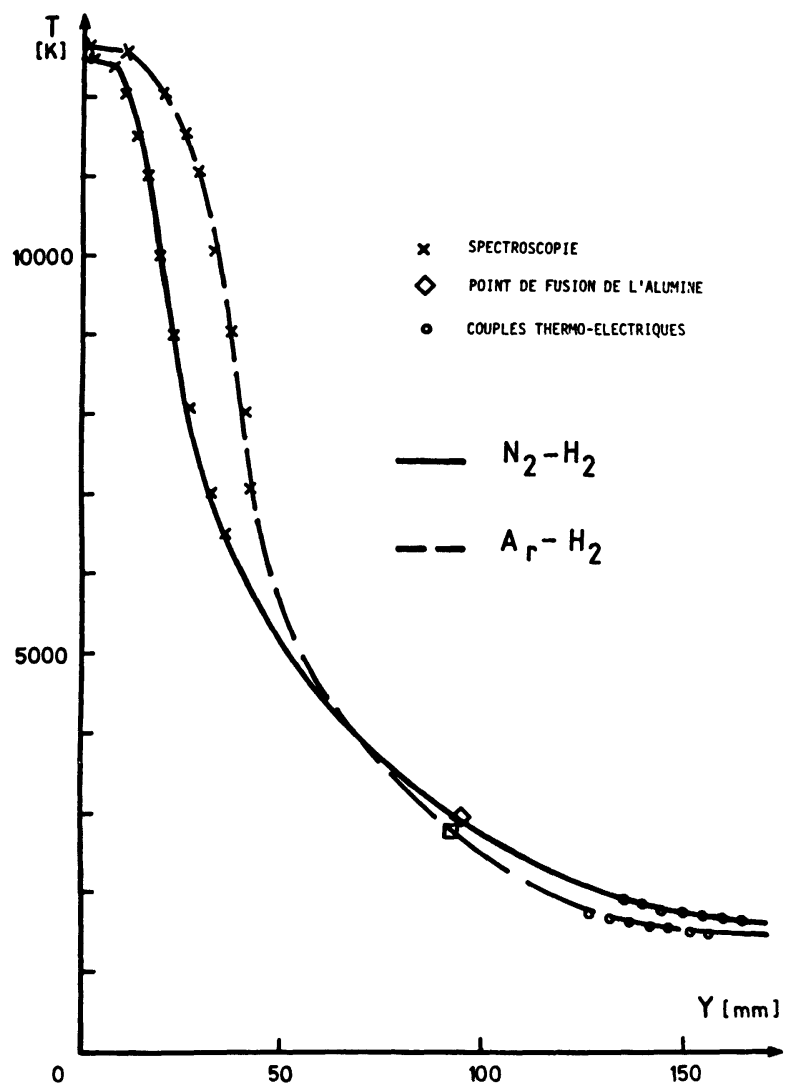

Fig. 10. - Evolution axiale de la température des plasmas azotehydrogène et argon-hydrogène.

[Axial profile of the $\mathrm{N}_{2}-\mathrm{H}_{2}$ and $\mathrm{Ar}-\mathrm{H}_{2}$ temperature.] 
tuyère. La vitesse d'injection du gaz dans cette région est alors déterminée en comparant les profils de vitesses calculés et mesurés pour des particules de différentes granulométries [6]. D'une façon générale, les vitesses du jet de plasma $\mathrm{N}_{2}-\mathrm{H}_{2}$, dans nos conditions de travail, sont plus élevées que celle du jet de plasma $\mathrm{Ar}-\mathrm{H}_{2}$. Ceci est dû, d'une part, à la viscosité moins élevée du mélange $\mathrm{N}_{2}-\mathrm{H}_{2}$ et, d'autre part, à une tuyère de diamètre inférieur pour ce mélange.

La fusion des particules étant fonction du transfert thermique plasma-particule, de la trajectoire suivie par les particules dans le plasma et du temps de séjour des particules dans le plasma, en projection, on travaille généralement avec des plasmas très stables d'argon auxquels on ajoute de l'hydrogène afin de bénéficier de l'augmentation notable de la conductibilité thermique moyenne du plasma dès $5000 \mathrm{~K}$ et d'obtenir des vitesses d'écoulement relativement basses (du fait de la grande viscosité de l'argon) permettant un temps de séjour suffisant pour la fusion des particules. En mesurant la température et la vitesse du jet de plasma pour différents débits de gaz et différentes caractéristiques géométriques de la tuyère, on peut déterminer le meilleur compromis pour avoir une température supérieure à $5000 \mathrm{~K}$ dans la plus grande partie du jet, une vitesse d'écoulement réduite et un rendement de générateur élevé. Par ailleurs l'azote étant un gaz nettement moins cher que l'argon, nous avons recherché les mêmes conditions d'optimisation pour un générateur fonctionnant avec un mélange azote-hydrogène : cela nécessite l'emploi d'un générateur avec un diamètre de tuyère inférieur à celui utilisé pour le mélange argon-hydrogène et à un débit de gaz plus faible.

2.3 Le fluX Des PaRTicules. - Le jet de plasma étant très hétérogène, les particules peuvent subir des traitements thermiques très différents selon leurs trajectoires.

Compte tenu de l'importance des gradients radiaux des propriétés du plasma et de la viscosité élevée du gaz (près de 10 fois la viscosité du même gaz à la température ambiante [7]), les trajectoires des particules sont en grande partie conditionnées par la vitesse d'injection des particules dans le jet. Par exemple nous avons déterminé [6] avec des particules d'alumine de $18 \mu \mathrm{m} \pm 3 \mu \mathrm{m}$ que si les particules ont une vitesse de $25 \mathrm{~m} / \mathrm{s}$ dans un plasma $\mathrm{N}_{2}-\mathrm{H}_{2}$ et de $32 \mathrm{~m} / \mathrm{s}$ dans un plasma $\mathrm{Ar}-\mathrm{H}_{2}$, elles pénètrent au cœur du jet de plasma et suivent pratiquement une trajectoire axiale. Pour des vitesses inférieures elles ne pénètrent pas complètement et restent en périphérie du jet et pour des vitesses supérieures elles traversent le jet de plasma et ont encore des trajectoires périphériques. Il est donc fondamental pour la qualité des dépôts d'optimiser les vitesses d'injection des particules pour chaque granulométrie afin qu'elles aient une trajectoire quasiaxiale et que la majorité d'entre elles passe dans les zones les plus chaudes du jet, comme le montre la figure 11 qui représente les courbes d'isoflux de particules d'alumine de $18 \mu \mathrm{m}$ de diamètre injectées dans un plasma $\mathrm{N}_{2}-\mathrm{H}_{2}$.

Cette vitesse optimale correspond à la meilleure répartition du jet de poudre dans le plasma, c'est-àdire à un jet de particules symétrique et peu divergent.

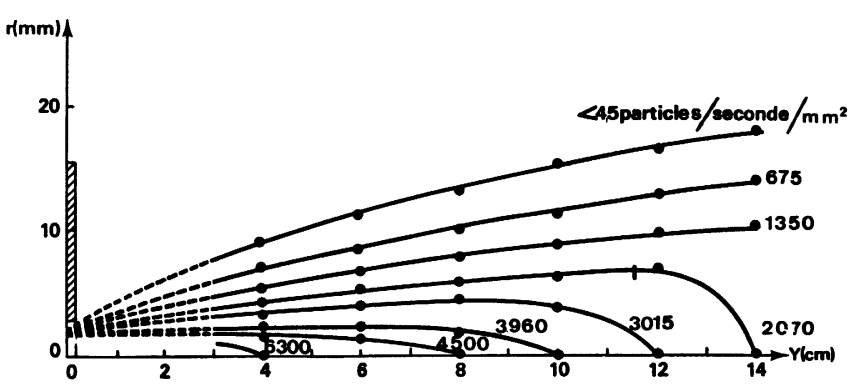

Fig. 11. - Courbes d'isoflux de particules de $18 \mu \mathrm{m}$ dans $\mu$ net de plasma $\mathrm{N}_{2}-\mathrm{H}_{2}$.

[Isoflux contours of $18 \mu \mathrm{m}$ particles in a $\mathrm{N}_{2}-\mathrm{H}_{2}$ plasma jet.]

2.4 LA FORMATION DU DÉPOT. - La projection plasma consiste à fondre le matériau de revêtement introduit sous forme pulvérulente dans le jet de plasma et à le projeter à grande vitesse sur l'objet à revêtir. Pour les matériaux céramiques, l'accrochage est essentiellement mécanique : les particules liquides s'accrochent sur le support et constituent, par apports successifs, un revêtement de structure lamellaire (Fig. 12).

Ceci est dû au fait que le temps de refroidissement moyen d'une particule est de l'ordre de $10 \mu$ s et que statistiquement une particule s'écrase au même point toutes les $0,1 \mathrm{~s}$. Chaque particule vient donc s'écraser sur une surface solide et il est essentiel pour la qualité du dépôt qu'elle s'écrase le mieux possible et que la goutte soit la moins visqueuse possible [8].

Lorsqu'une particule frappe la cible son degré de déformation (rapport du diamètre de la goutte étalée à son diamètre initial) dépend de la viscosité, c'est-à-dire essentiellement de sa température, de son énergie cinétique, de sa tension de surface et des cinétiques de cristallisation [8]. La température de la particule au moment de l'impact est le facteur prépondérant pour le bon étalement de la particule, à moins que sa température ne soit très supérieure à la température de fusion. Dans ce cas, la vitesse à l'impact devient le facteur principal.

Le refroidissement très rapide à partir de la phase liquide est caractéristique de la projection plasma; il peut en résulter la formation de phases métastables. L'exemple le plus classique est la projection de l'alumine avec la formation de phases métastables de type spinel de préférence à la structure $\alpha$ stable à basse température. Ceci est certainement dû à l'énergie d'activation pour la nucléation plus faible pour la structure spinelle lorsque le liquide est refroidi bien en dessous du point d'équilibre lors de la trempe rapide. Les qualités du revêtement dépendent donc 


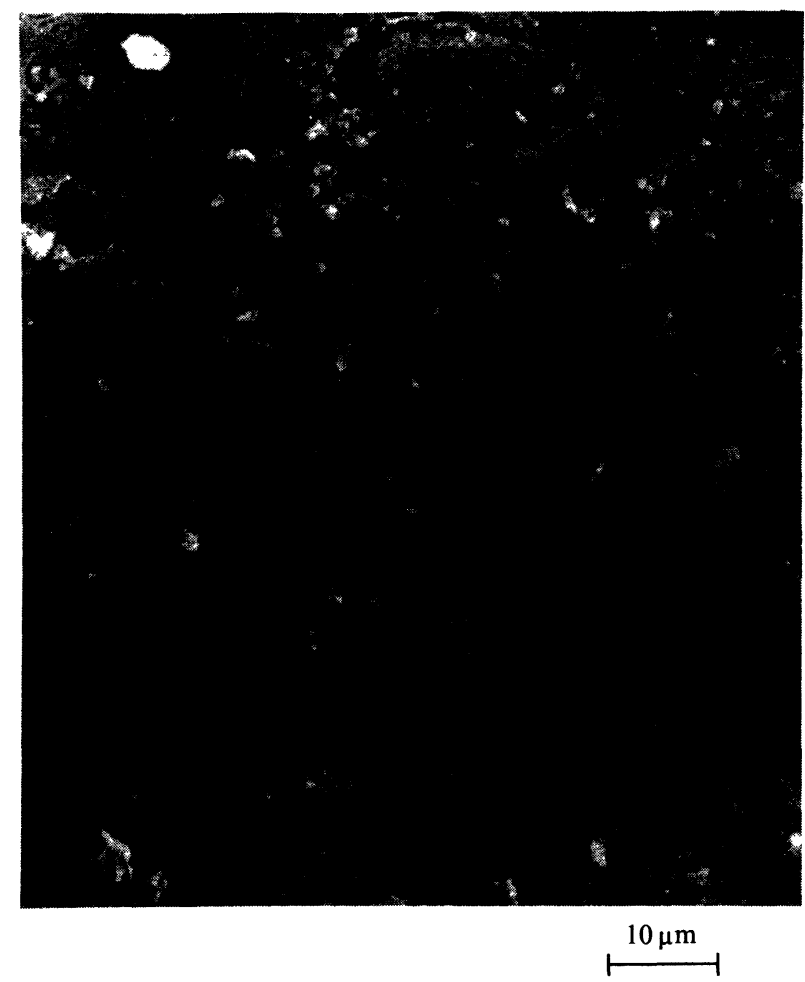

Fig. 12. - Micrographie de la structure lamellaire d'un revêtement d'alumine.

[SEM picture of the lamellar structure of an alumina coating.]

fortement des mécanismes de déformation, de solidification et d'adhérence des gouttelettes frappant la surface à recouvrir.

3. Résultats expérimentaux : corrélations entre l'obtention de dépôts denses d'alumine $\gamma$ et les conditions opératoires de projection. - A partir des mesures des vitesses et des températures des particules dans le jet de plasma, il est possible d'optimiser les conditions de fonctionnement de l'installation de projection pour obtenir des dépôts denses d'alumine en phase $\gamma$ : l'obtention de revêtements en phase métastable $\gamma$ dépend essentiellement de la température des particules à l'impact sur la cible et de leurs conditions de refroidissement, alors que la porosité du dépôt est liée principalement à l'énergie cinétique et à la viscosité des particules.

Pour un matériau donné, les principaux paramètres de fonctionnement à étudier sont la géométrie de tir, la puissance électrique fournie à l'arc, la nature du gaz plasmagène, la morphologie de la poudre, les conditions de refroidissement (Fig. 13).

3.1 GÉOMÉTRIE DE TIR. - L'évolution axiale de la vitesse et de la température est donnée à la figure 14 pour des particules de poudre DGI 5 de $18 \mu \mathrm{m}$, traitées dans un plasma $\mathrm{Ar}-\mathrm{H}_{2}$ à $29 \mathrm{~kW}$.

La réalisation de dépôts denses nécessitant que les particules s'écrasent sur la cible à l'état liquide avec

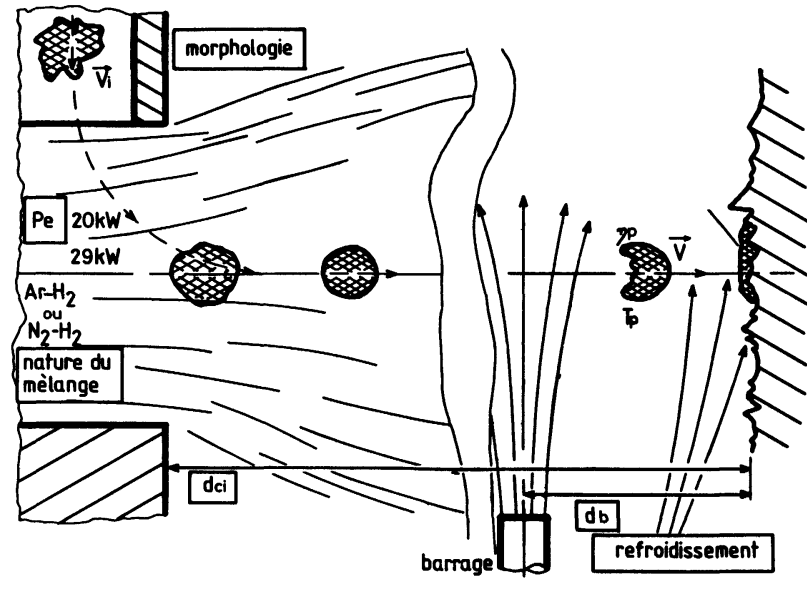

Fig. 13. - Principaux paramètres influençant la vitesse et la température des particules.

[Main parameters which influence the particles velocity and temperature.]

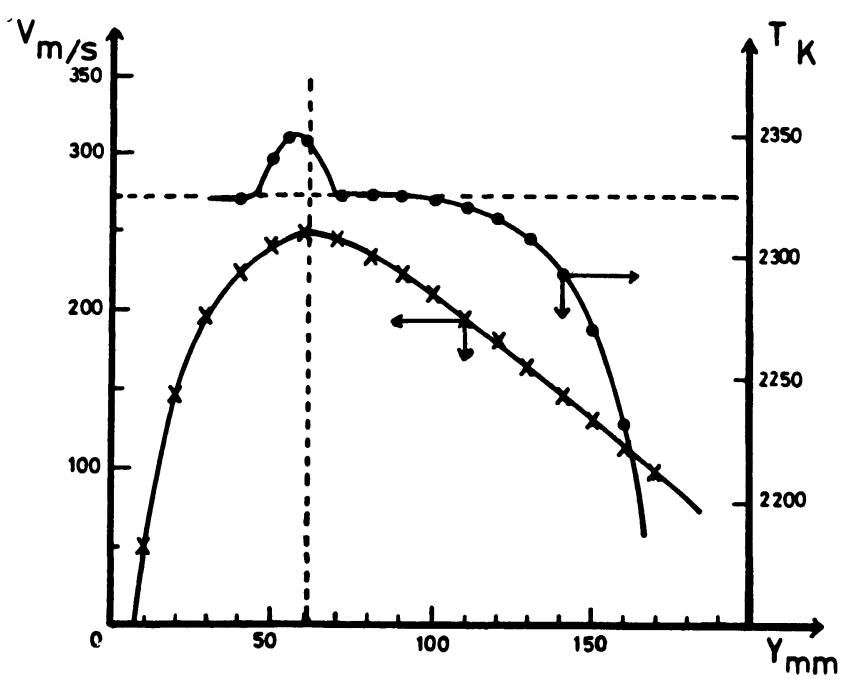

Fig. 14. - Evolution axiale de la vitesse et de la température des particules de $18 \mu \mathrm{m}$ dans un jet de plasma $\mathrm{N}_{2}-\mathrm{H}_{2}$.

[Axial profile of the temperature and velocity of $18 \mu \mathrm{m}$ particles in a $\mathrm{N}_{2}-\mathrm{H}_{2}$ plasma jet.]

une énergie cinétique maximale et une viscosité faible, la cible doit être placée à l'abscisse correspondant à la vitesse et à la température maximales des particules. D'après la figure 14 la distance torchecible doit être de $55 \mathrm{~mm}$ dans ce cas.

Si les deux maxima ne coïncident pas il faut déterminer le meilleur compromis, la température étant le facteur prépondérant.

3.2 Puissance ÉlectriQue fourNie a L'ARC. L'élévation de la puissance électrique fournie à l'arc se traduit essentiellement par une augmentation de la vitesse des particules et un déplacement du maximum de vitesse comme le montrent les courbes de la figure 15:P=20 kW, $V_{\max }=240 \mathrm{~m} / \mathrm{s}$ à $50 \mathrm{~mm}$; 


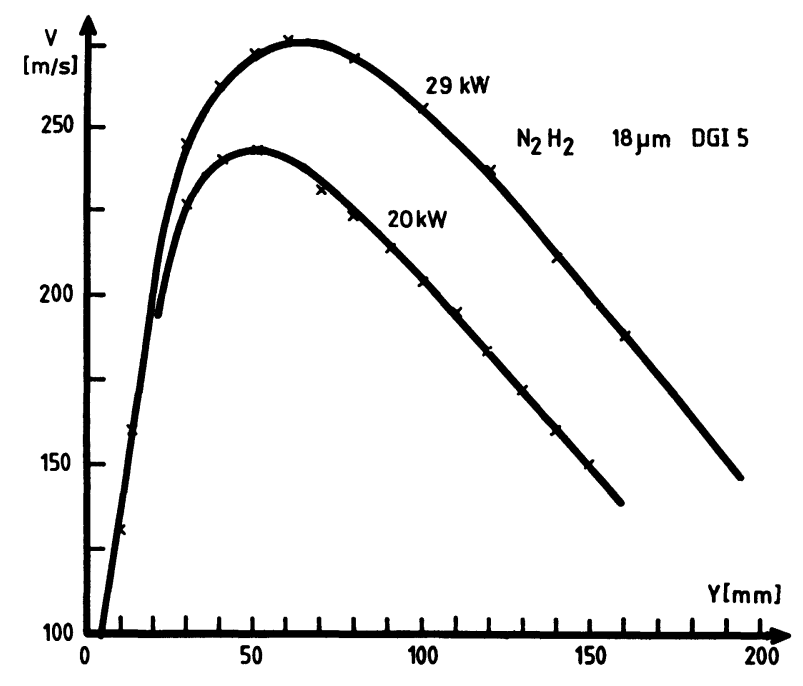

Fig. 15. - Evolution axiale de la vitesse des particules de $18 \mu \mathrm{m}$ dans un jet de plasma $\mathrm{N}_{2}-\mathrm{H}_{2}$ à une puissance de 20 et $29 \mathrm{~kW}$.

[Axial profile of $18 \mu \mathrm{m}$ particles velocity in a $\mathrm{N}_{2}-\mathrm{H}_{2}$ plasma jet for 20 and $29 \mathrm{~kW}$ electrical power.]

$P=29 \mathrm{~kW}, V_{\max }=275 \mathrm{~m} / \mathrm{s}$ à $60 \mathrm{~mm}$ pour un plasma $\mathrm{N}_{2}-\mathrm{H}_{2}$. Mais corollairement, le temps de séjour des particules dans les zones chaudes du plasma diminue. L'augmentation de la puissance électrique n'entraîne qu'une faible élévation de la température des particules (Fig. 16), la diminution du temps de séjour compensant l'augmentation de la densité d'énergie du plasma. Sauf pour un plasma $\mathrm{N}_{2}-\mathrm{H}_{2}$ à $20 \mathrm{~kW}$, la

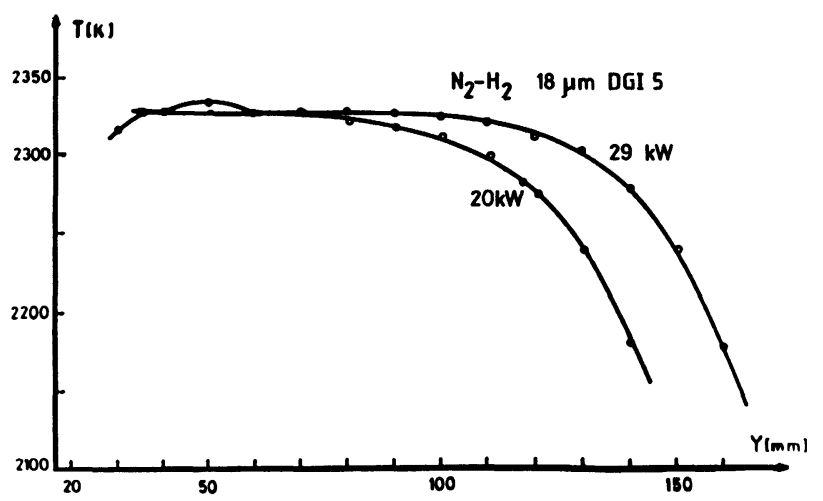

Fig. 16. - Evolution axiale de la température des particules de $18 \mu \mathrm{m}$ dans un jet de plasma $\mathrm{H}_{2}-\mathrm{N}_{2}$ à une puissance de 20 et $29 \mathrm{~kW}$.

[Axial profile of $18 \mu \mathrm{m}$ particles temperature in a $\mathrm{N}_{2}-\mathrm{H}_{2}$ plasma jet for 20 and $29 \mathrm{~kW}$ electrical power.]

proportion de phase $\alpha$ dans les dépôts reste sensiblement constante et inférieure à $2 \%$. Ce seuil semble difficile à abaisser compte tenu des cinétiques de transformation $\gamma \rightarrow \alpha[8]$. Quels que soient le gaz plasmagène et la poudre utilisés, une augmentation de la puissance électrique fournie à l'arc au-dessus d'un certain seuil (correspondant à la fusion complète des particules) n'améliore pas de façon notable la proportion d'alumine métastable obtenue. Par contre, elle entraîne une légère diminution de la porosité et du diamètre moyen des pores (tableau I) liée à l'augmentation de l'énergie cinétique.

Tableau I.

\begin{tabular}{|c|c|c|c|}
\hline Puissance & Poudre & $\begin{array}{c}\mathbf{R X} \\
\% \alpha /(\alpha+\gamma)\end{array}$ & $\begin{array}{c}\text { Porosité } \\
\%\end{array}$ \\
\hline $\begin{array}{ll}29 \mathrm{~kW} & \\
& 100 \mathrm{~V} \\
& 290 \mathrm{~A}\end{array}$ & $\begin{array}{l}\text { A } 125 \\
\text { GE } 1 \\
\text { DGI } 5\end{array}$ & $\begin{array}{l}4 \\
6 \\
4\end{array}$ & $\begin{array}{l}8,5 \\
9 \\
9\end{array}$ \\
\hline $\begin{array}{rr}20 \mathrm{~kW} & \\
& 100 \mathrm{~V} \\
& 200 \mathrm{~A}\end{array}$ & $\begin{array}{l}\text { A } 125 \\
\text { GE } 1 \\
\text { DGI } 5\end{array}$ & $\begin{array}{l}5 \\
8 \\
5,5\end{array}$ & $\begin{array}{l}10,5 \\
11 \\
11\end{array}$ \\
\hline
\end{tabular}

3.3 Nature du Gaz Plasmagène. - Pour nos conditions de travail, le plasma $\mathrm{Ar}-\mathrm{H}_{2}$ est plus lent que le plasma $\mathrm{N}_{2}-\mathrm{H}_{2}$. Les vitesses atteintes par les particules sont plus faibles dans le $1^{\mathrm{er}}$ cas (Fig. 17) mais leur temps de séjour dans les zones chaudes est plus élevé $(20 \%)$ et leur échauffement plus important (Fig. 18). Les particules ainsi traitées s'écrasent sur la cible avec une viscosité plus faible ce qui fait plus que compenser la perte en énergie cinétique et leur étalement est meilleur.

La porosité des dépôts est plus faible : $3 \%$ au lieu de $9 \%$. D'autre part le dard du jet de plasma $\mathrm{Ar}-\mathrm{H}_{2}$ est plus long et le panache plus expansé ce qui conduit à un traitement plus homogène des particules. La proportion des particules traitées de façon optimale est plus élevée et la teneur en $\alpha$ dans le dépôt plus basse : $1 \%$ au lieu de $6 \%$.

3.4 MORPHOLOGIE DE LA POUDRE. - La poudre DGI 5 , monocristalline, atteint une température de surface légèrement plus élevée que celle des poudres constituées d'agglomérats (Fig. 19).

En effet, pour les agglomérats, la diffusion de la chaleur est beaucoup plus difficile d'un cristallite à l'autre. La poudre GE 1 dont les cristallites élémentaires sont de $1,5 \mu \mathrm{m}$, a une température de surface légèrement supérieure à celle de la poudre $\mathrm{A} 125$ dont les cristallites élémentaires sont de $0,01 \mu \mathrm{m}$. Les transferts de chaleur sont d'autant meilleurs que la surface spécifique des particules est plus faible : 


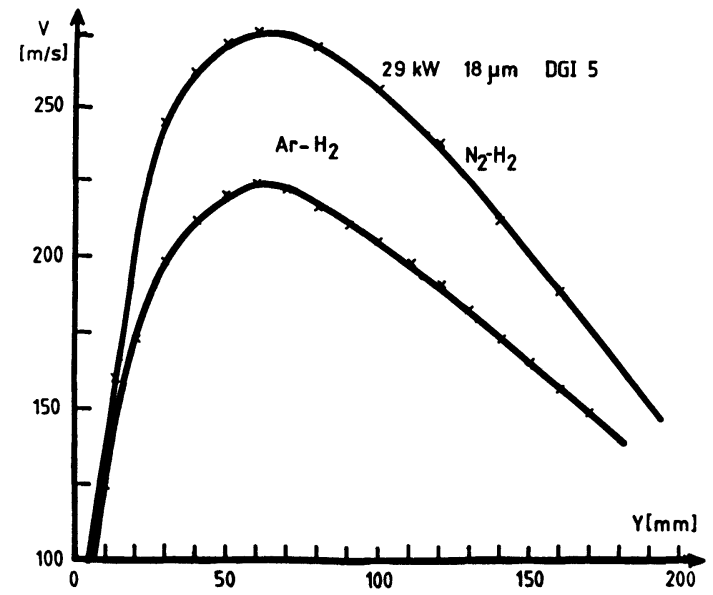

Fig. 17. - Evolution axiale de la vitesse des particules de $18 \mu \mathrm{m}$ dans des jets de plasma $\mathrm{N}_{2}-\mathrm{H}_{2}$ et $\mathrm{Ar}-\mathrm{H}_{2}$ à une puissance de $29 \mathrm{~kW}$.

[Axial profile of $18 \mu \mathrm{m}$ particles velocity in a $\mathrm{N}_{2}-\mathrm{H}_{2}$ and a $\mathrm{Ar}-\mathrm{H}_{2}$ plasma jet.]

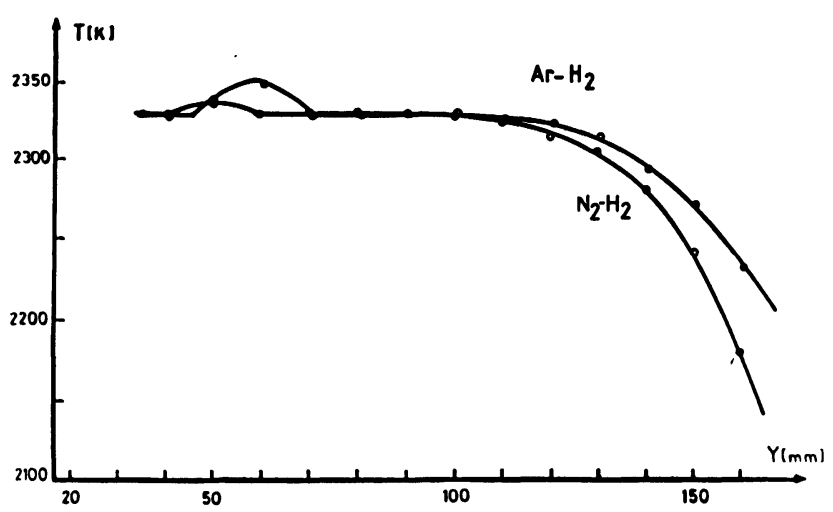

Fig. 18. - Evolution axiale de la température des particules de $18 \mu \mathrm{m}$ dans des jets de plasma $\mathrm{N}_{2}-\mathrm{H}_{2}$ et $\mathrm{Ar}-\mathrm{H}_{2}$ à $29 \mathrm{~kW}$.

[Axial profile of $18 \mu \mathrm{m}$ particles temperature in a $\mathrm{N}_{2}-\mathrm{H}_{2}$ and $\mathrm{Ar}-\mathrm{H}_{2}$ plasma jet.]

$1 \mathrm{~m}^{2} / \mathrm{g}$ pour GE 1 contre $115 \mathrm{~m}^{2} / \mathrm{g}$ pour A 125 . Cela se traduit toutefois par une porosité légèrement plus faible dans le cas des dépôts réalisés avec la poudre monocristalline.

3.5 REFROIDISSEMENT DE LA CIBLE. - Les cibles sont généralement maintenues à une température de l'ordre de $320 \mathrm{~K}$, par un jet d'air comprimé.

Une élévation de température de la cible en favorisant l'étalement des particules entraîne une diminu-

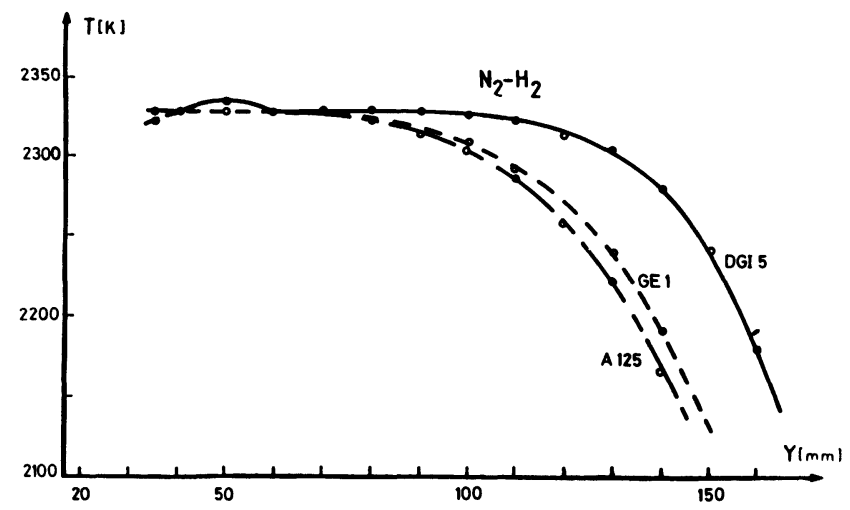

Fig. 19. - Influence de la morphologie de la poudre sur la température des particules dans un jet de plasma $\mathrm{N}_{2}-\mathrm{H}_{2}$ à $29 \mathrm{~kW}$.

[Influence of powder morphology on particles temperature in a $\mathrm{N}_{2}-\mathrm{H}_{2}$ plasma jet.]

tion de la porosité du revêtement. En projetant sur une cible non refroidie, le dépôt réalisé est constitué d'alumine en phase $\alpha$ et d'alumine métastable lorsque la température est de l'ordre de $1300 \mathrm{~K}$ et essentiellement d'alumine $\alpha$ lorsque la température est de $1500 \mathrm{~K}$, la trempe subie par les particules n'étant plus assez sévère pour figer la phase métastable.

4. Conclusion. - Les caractéristiques des dépôts obtenus par projection au chalumeau à plasma d'arc (microstructure, porosité, état cristallographique...) dépendent des conditions de réalisation de ces dépôts. Les paramètres de réglage d'une installation sont nombreux et souvent inter-dépendants. Pour analyser les relations entre ces paramètres et la morphologie des dépôts, nous avons déterminé les caractéristiques de vitesse et de température du plasma ainsi que la vitesse et la température des particules tout au long de leurs trajectoires depuis la sortie de tuyère du chalumeau.

Cette étude a permis d'optimiser les conditions de fonctionnement de notre installation dans le cas de la projection de poudres d'alumine en vue d'obtenir des dépôts denses en phase $\gamma$ métastable. Par ailleurs, en mettant en relief l'importance des paramètres microscopiques du plasma, elle devrait aider à modéliser le procédé de projection afin de déterminer les conditions optimales pour des poudres de matériau divers quelle que soit leur granulométrie. De premiers résultats ont déjà été obtenus en ce sens [9].

\section{Bibliographie}

[1] Brevet C.N.R.S. 124111 et additifs 168044, 7044678.

[2] Vardelle, A., Baronnet, J. M., Vardelle, M., Fauchais, P., IEEE Trans. Plasma Science. PS 8 (1980) 417.

[3] Kruszewska, B., Lesinski, J., Revue Phys. Appl. 12 (1977) 1209.

[4] Hinze, J. O., Prog. Heat Mass Transfer 6 (1972) 433.

[5] Bourdin, E., Fauchais, P., Boulos, M., Proc. Heat Transfer Symposium, octobre (1980) Edmonton, Canada.
[6] VARDELle, M., Thèse $3^{e}$ cycle, Université de Limoges (1980).

[7] Capitelli, M., Gorse, C., Fauchais, P., J. Physique 38 (1977) 653.

[8] McPherson, R., J. Mater. Sci. 8 (1973) 851.

[9] Bourdin, E., Vardelle, A., Vardelle, M., Fauchais, P., Proc. 5th International Symposium on plasma chemistry. Edinburgh, août (1981). 